\title{
The Impact of Question Format, Context, and Content on Survey Answers in Early and Late Adolescence
}

\author{
Nadine Diersch ${ }^{1}$ and Eva Walther ${ }^{2}$
}

\begin{abstract}
Self-reports in surveys are often influenced by the presented question format and question context. Much less is known about how these effects influence the answers of younger survey respondents. The present study investigated how variations in response format, answer scale frequency, and question order influence self-reports of two age groups: younger (11-13 years old) and older (16-18 years old) adolescents. In addition, the impact of the respondents' level of familiarity with the question content was taken into account. Results indicated that younger adolescents are more strongly influenced by the presented question format and context than older adolescents. This, however, was dependent on the particular question content, implying that response effects are more pronounced when questions deal with issues that lie outside of the respondents' field of experience. Implications of these findings in survey research with younger respondents are discussed.
\end{abstract}

Key words: Attitude judgments; question order effects; social influence; survey methodology; younger survey respondents.

\section{Introduction}

Self-reports are often the method of choice in the social and behavioral sciences to collect information about people's attitudes, beliefs, intentions, and behaviors (Krosnick 1999; Schwarz 1999). The age group between 5 and 15 years of age is of growing importance in public opinion and consumer research due to their increasing purchasing power and influence on families' purchasing decisions (De Leeuw et al. 2004; Heinzel 2000; McNeal 1992). In large-scale surveys like the international "EU Kids Online" survey, for example, thousands of children and adolescents aged 9 to 16 are regularly asked about their living situation, values, behaviors, and future plans (cf., Livingstone et al. 2012). Given the challenges that the inclusion of younger age groups poses to survey research, Scott (2008) concluded in her review on children as respondents that "improved data about children are essential in a society where children's role as consumers and citizens is being taken increasingly seriously in the economy, in law, and in social policy" (Scott 2008, 103). Thus, a better understanding of whether younger respondents interpret the questions as

1 Aging \& Cognition Research Group, German Center for Neurodegenerative Diseases (DZNE), Magdeburg, Germany, and Department of Psychology, Chemnitz University of Technology, Chemnitz, Germany. Email: nadine.diersch@dzne.de

${ }^{2}$ Department of Psychology, University of Trier, Universitätsring 15, 54296 Trier, Germany. Email: walther@uni-trier.de

Acknowledgments: The authors thank the pupils of the "Diesterweg-Gymnasium" in Plauen, Germany, for donating their time to take part in the study, Karin Zahradnik and Rüdiger Stumm for their support in conducting the survey at this school, and Norbert Schwarz for helpful comments on an earlier version of this article. 
intended by the (adult) researcher is needed in order to collect meaningful data in these age groups and interpret the results accordingly.

In general, answering a survey question is a complex cognitive process consisting of several tasks, ranging from understanding the intended meaning of the question to retrieving relevant information from memory, forming an appropriate judgment, and finally translating it into an answer provided by the questionnaire (Tourangeau and Rasinski 1988). It has been suggested that this answer process might be understood as a form of conversation (see Schwarz 1999; Strack and Schwarz 2007 for reviews). In contrast to a natural conversation, however, direct feedback from the conversation partner (i.e., the researcher) is lacking in standardized surveys, which in turn might lead to a greater reliance on contextual cues in order to infer the intended meaning of a question. Thus, social or contextual influences can affect any stage of the answer process, as indicated by a vast amount of research (e.g., Schuman and Presser 1981; Schwarz 1999; Schwarz and Bohner 2001; Schwarz and Hippler 1995; Schwarz and Oyserman 2001; Strack and Schwarz 2007). The results of these studies suggest that the interpretation and categorization of a question may be influenced strongly by contextually provided cues such as the respective question wording, format, and order (cf. Gawronski and Cesario 2013). However, the sensitivity to the context may be modulated by the particular topic in question and the respondents' level of familiarity and knowledge about it (cf. Festinger 1954). In line with this, Bickart (1992) provided evidence that brand evaluations are influenced more strongly by the presented question context in respondents who possess low levels of category-specific knowledge as compared to highly experienced respondents.

Although these response effects are well documented in adult respondents, much less is known regarding such effects in younger survey respondents. Because the answer process in a survey draws on several cognitive resources such as attention and memory, agerelated differences in cognitive functioning are likely to result in different answers to the very same question as a function of age (see Knäuper et al. 2007; Schwarz 2003 for examples of survey research with respondents aged 60 and older). In accordance with cognitive-developmental theories (see Goswami 2010 for an overview), it is usually assumed that data reliability increases with age and cognitive development (cf. Borgers et al. 2000; Fallon Jr. and Schwab-Stone 1994; Reynolds 1993). For example, Borgers et al. (2000) concluded in their secondary analysis of existing data with different age groups that children around the age of eleven are able to give relatively consistent answers although they are very context sensitive and may invest less effort into the question-answer process if they are not interested in the topic in question or if the meaning of the question is somewhat unclear to them. In their review of empirical evidence about developmental influences on the ability to answer survey questions, De Leeuw et al. (2004) pointed out that cognitive functioning (e.g., memory) is comparable to that of adult respondents at the end of middle childhood ( 7 to 12 years of age), but that suggestibility resulting from social and motivational factors might influence self-reports of this age group. Children, more so than adults, tend towards answers they perceive as socially desirable or that reflect their own subjective wishes (Kränzl-Nagl and Wilk 2000; Reynolds 1993). Children below the age of twelve are generally more susceptible to social influence than adults, particularly when they receive information from authorities (Ceci et al. 1987; Warren and Lane 1995). 
Adolescents at the age of 16 , by contrast, are assumed to be able to answer survey questions similarly to adult respondents (De Leeuw et al. 2004). Thus, younger respondents might also take the presented question format and context into account when answering a survey, especially when they are not very familiar with the topic in question. Below the age of 16, social desirability concerns and satisficing, that is, the reliance on simple heuristics to give an answer due to a lack of motivation, might additionally bias the answers in a survey (Borgers et al. 2000; De Leeuw et al. 2004).

In the following, four types of response effects will be outlined that are well documented in the literature on survey research with adult respondents. Next, studies will be reviewed providing a first indication of how younger survey respondents might be influenced by the presented question format and context. Finally, an overview of the present study will be provided.

\subsection{Effects of Question Format and Context on Self-Reports in a Survey}

Adult respondents often react differently when they are offered a specified set of response alternatives compared with a situation in which no response alternatives are given (e.g., Kane and Schuman 1991; Schuman and Presser 1981; Schwarz 1999; Schwarz and Oyserman 2001; Strack and Schwarz 2007). In an open response format, relevant information has to be retrieved from memory in a first step. In the next step, a decision has to be made as to which information is relevant in the context of the question. In a closed response format, the first step can be skipped, resulting in more responses on average. Moreover, predefined response alternatives might indicate which level or type of response is considered appropriate in the context of the survey. In addition, several studies have shown that the frequency of answer scales and their reference points may be used as a frame of reference to infer the intended meaning of a question, which in turn might influence judgments about the frequency of own behaviors (e.g., Schwarz 1999; Schwarz and Bienias 1990; Schwarz et al. 1985; Schwarz and Oyserman 2001; Strack and Schwarz 2007).

Not only reports about actual behaviors are highly context-dependent; the same is true of reports about personal opinions, interests, and attitudes. This implies that attitudes are often "constructed on the spot" (Schwarz and Bohner 2001, 442). Numerous studies have shown that preceding questions influence the answers to subsequent questions (e.g., Bishop 1987; Krosnick 1991; Krosnick and Alwin 1987; Schuman and Presser 1981; Schwarz 1999; Schwarz and Hippler 1995, Strack 1992; Strack and Schwarz 2007; Tourangeau et al. 1989). According to the norm of evenhandedness, respondents answer two questions that are similar in some important aspect in an evenhanded way in order to appear consistent (Schuman and Presser 1981). Thus, a personal preference might be adjusted to an answer on a preceding question if this norm is evoked. Comparable mechanisms operate when respondents have to answer certain filter questions before or after they report personal attitudes and opinions on the same topic (Strack 1994). These questions, whether they are related to own behaviors or knowledge about the topic in question, also make information available that might shape the answers to following questions (cf., Bishop 1987; Bishop et al. 1983; Schwarz et al. 1991). Self-perception and self-evaluation may be altered by answering a filter question, resulting in changes 
in the focus of attention afterwards in order to reaffirm the self-concept (Martin and Harlow 1992).

\subsection{Effects of Question Format and Context on Self-Reports of Younger Survey Respondents}

To date, there are only very few studies that investigate the impact of question format and context on the answers of younger respondents in a survey. For example, Borgers et al. (2004) provided evidence that the reliability of self-reported self-esteem and well-being in children and younger adolescents between 8 and 16 years of age is modulated by the number of presented response options, the usage of a neutral midpoint, and negatively formulated questions. It was shown that the internal consistency of the self-reports increases with increasing numbers of response options on a rating scale but decreases if more than six response options are presented. The consistency over time, however, showed the reverse pattern of results. Negatively formulated questions did not influence the reliability, although the answers generally differed between negatively and positively formulated questions. Possible answer differences within the sample that encompassed a relatively large age range, however, were not taken into account.

Fuchs (2005) examined the influence of question format and context on self-reports of different younger age groups in several independent studies. The respondents' cognitivedevelopmental status was additionally assessed by means of self-reported educational achievement. The results showed that the impact of response order, response scale, and numeric values associated with response alternatives was higher in children and younger adolescents (aged 10 to 13 in Study 1, aged 13 to 15 in Study 2) compared to older adolescents (aged 14 to 17 in Study 1, aged 16 to 17 in Study 2) and adults (aged 18 and older in all studies). In addition, the effects tended to be more pronounced in respondents with poor or intermediate educational achievement. At the same time, younger respondents (aged 13 to 15 and aged 16 to 17) were less affected by the presented question order than older respondents (aged 18 and older), indicating that information from preceding questions was not taken into account in the formation of a judgment on following questions. However, correlations between the different questions, the order of which was manipulated, were not reported. The analysis focused on relative differences in answers to the single questions as a function of question order. In general, it was concluded that limited cognitive skills may result in an incomplete understanding of a given question and an insufficient retrieval of relevant information, resulting in a less sophisticated question-answer process in younger survey respondents. Notably, the composition of the single age groups as well as the content of the critical questions varied across the single experiments, making it difficult to compare the data across experiments. In a follow-up study, standard tests of cognitive functioning (e.g., vocabulary and working memory tests) were applied in a sample of children between 8 and 14 years of age to examine the relation between age, cognitive functioning, and respondent problems (e.g., inadequate answers, uncertainty) in a face-to-face interview setting (Fuchs 2009). The results confirmed that increasing age leads to fewer problems in the question-answer process. This, however, was largely independent from differences in cognitive functioning. Thus, one might speculate that other factors such as social and/or motivational factors might 
contribute to the observed answer differences in younger survey respondents. As outlined above, children and younger adolescents might be more likely to construct certain attitudes "on the spot", resulting in answers that are based to a larger extent on the information available at the time they are asked, due to their higher suggestibility and satisficing strategies (De Leeuw et al. 2004).

\subsection{Overview of the Present Study}

The present study aimed at investigating how variations in question format and context affect the answers of two age groups, younger (11-13 years old) and older (16-18 years old) adolescents, when they are interviewed in a survey setting. The two age groups were chosen because they represent the lower and upper end of adolescence. Based on previous research, we assumed that social desirability concerns and motivational factors would become more evident in the younger age group, whereas cognitive functioning would be relatively comparable between the two groups (cf. De Leeuw et al. 2004). Going beyond previous research, we also addressed the impact of the particular question content on the answers of the two age groups. More specifically, we examined to what extent the subjective experience with a certain question domain would shape the potentially biasing influences of contextual cues provided by the questionnaire. In addition, by using a relatively short questionnaire that incorporated a number of aspects, we aimed to resemble a typical situation in survey research, where many data need to be gathered while keeping questionnaire length at a reasonable level in order to reduce dropouts. We developed two versions of a questionnaire in which four response effects (i.e., open vs. closed response formats, frequency of answer scales, question order in attitude judgments, and question order of filter questions in combination with attitude judgments) were varied within two different topics: environmental protection and sports activities. The two topics were selected because we assumed that respondents have less experience with the former as compared to the latter. As a consequence, reports about environmental protection activities should be more affected by social desirability concerns than sports activities, and if younger adolescents are more susceptible to social influence than older adolescents this should be become evident within this topic.

We hypothesized that the difference between the open and closed response format is stronger in younger than in older adolescents. Younger adolescents might invest less effort in the question-answer process and consequently rely more on predefined response sets than older adolescents, especially if the topic in question is rather unfamiliar and less interesting to them (cf. Borgers et al. 2000). In addition, we expected that younger adolescents would be more strongly influenced by the frequency of answer scale than older adolescents because they tend more towards socially desirable answers due to a higher suggestibility (cf., De Leeuw et al. 2004; Kränzl-Nagl and Wilk 2000). Preceding questions might shape subsequent attitude judgments more in younger than in older adolescents because they are more context sensitive, especially when they have not already formed a firm attitude about the topic in question (cf. Borgers et al. 2000). Similarly, attitude judgments might be more strongly influenced in younger than in older adolescents by the success or failure answering a filter question beforehand due to information about own relevant behaviors that is brought to mind. 


\section{Method}

\subsection{Participants}

The survey was completed by 188 pupils from a German secondary school following the survey's approval by the local school board. The sample consisted of two age groups: younger adolescents $\left(n=104,54\right.$ female, $M_{\text {age }}=12.2$ (mean), SD $=0.72$ (standard deviation), age range: $11-13$ years) and older adolescents $\left(n=84,39\right.$ female, $M_{\text {age }}=16.9$, $\mathrm{SD}=0.61$, age range: $16-18$ years), and was largely homogeneous in terms of academic performance. All of them aspired to the highest educational qualification available in the German school system and when asked which school grade they achieved most frequently, $88.8 \%$ of the pupils reported to receive good or satisfactory grades on average. For respondents under the age of $18(n=179)$, informed consent was obtained from their parents. Three pupils whose parents refused permission were excluded from participation. Additional written informed consent was obtained from the respondents themselves.

\subsection{Design and Material}

Each age group received one of two questionnaire versions in which question format, frequency of answer scales, question order in attitude judgments, and order of filter questions in combination with attitude judgments were manipulated in a balanced order. Half of the questions in each questionnaire version referred to environmental protection activities and the other half to sports activities. In the section about environmental protection activities in one questionnaire version (Questionnaire A), for example, a certain question was presented in an open response format, whereas in the section about sports activities, a certain question was presented in a closed response format. In the other questionnaire version (Questionnaire B), the same questions were presented in the reverse format, that is, in a closed response format in the section about environmental protection activities and in an open response format in the section about sports activities. The remaining response effects were varied accordingly (see Table 1 for an overview of the questionnaire design). Special care was taken to word the question in an easy and understandable manner and to capture the characteristics of the two topics in an ecologically valid way. In the following, the implementation of each response effect within the two questionnaire sections will be outlined in detail.

\subsubsection{Open vs. Closed Response Format}

In order to investigate the influence of different response formats on the number and type of retrieved answers, a question in each thematic section was presented either in a closed response format or in an open response format. In the section about environmental protection activities, the question "Are you actively involved in saving the environment?", if yes, "How do you do that?" was presented with no answer alternatives in one questionnaire version, and with a list of twelve different answer alternatives (e.g., "I am a member of an environmental protection organization") in the other questionnaire version. With regard to sports activities, the question "Do you engage in sports?", if yes, "Which sports do you do?" was varied correspondingly with a list of 31 different sports activities or with no answer alternatives. 
Table 1. Variation of four response effects within two thematic sections in two questionnaire versions

\begin{tabular}{|c|c|c|}
\hline & Questionnaire A & Questionnaire B \\
\hline \multicolumn{3}{|l|}{ Response format } \\
\hline Environ. protection activities & Open & Closed \\
\hline Sports activities & Closed & Open \\
\hline \multicolumn{3}{|l|}{ Frequency of answer scale } \\
\hline Environ. protection activities & Low frequency & High frequency \\
\hline Sports activities & High frequency & Low frequency \\
\hline \multicolumn{3}{|c|}{ Question order in attitude judgments } \\
\hline Environ. protection activities & 1st pair: Specific - general & 1st pair: General - specific \\
\hline & 2nd pair: General - specific & 2nd pair: Specific - general \\
\hline & 3rd pair: Specific - general & 3rd pair: General - specific \\
\hline & 4th pair: General - specific & 4th pair: Specific - general \\
\hline Sports activities & $\begin{array}{l}4 \text { items about sports - } \\
4 \text { items about soccer }\end{array}$ & $\begin{array}{l}4 \text { items about soccer - } \\
4 \text { items about sports }\end{array}$ \\
\hline \multicolumn{3}{|l|}{ Order of filter questions } \\
\hline Environ. protection activities & After attitude judgments & Before attitude judgments \\
\hline Sports activities & Before attitude judgments & After attitude judgments \\
\hline
\end{tabular}

\subsubsection{Frequency of Answer Scales}

The influence of answer scale frequencies on reported behavioral frequencies was tested in the section about environmental protection activities in that participants were asked whether they sought information about issues of environmental protection, and if so, how often they did that on average. In one questionnaire version, this was accompanied by a low-frequency scale with five answer alternatives ranging from "Several times a week" to "Less than once a month". The high-frequency answer scale in the second questionnaire version comprised a reference period from "Several times a day" to "Less than once a week" with five answer alternatives (see Table 3 for item wording). Within the section about sports activities, the same two answer scales were used for the question "Do you engage in sports?", if yes, "How often do you do that?".

\subsubsection{Question Order in Attitude Judgments}

To test the impact of question order in attitude judgments, different items consisting of statements that either referred to a rather general aspect of the topic in question (i.e., how everybody should behave or the general attitude towards the topic) were used together with more specific statements referring to subordinate and personal aspects of the respective topic. In the section about environmental protection activities, four item pairs were presented in a varying order across the two questionnaire versions (see Table 2 for item wording). The overall order of the item pairs was kept constant. Every item pair was separated from the others by a "buffer" item about animal protection issues to reduce spillovers between the item pairs. In the section about sports activities, the question order was varied in blocks such that in one questionnaire version four items about sports in general preceded four items about soccer, whereas in the other questionnaire version the general items followed the specific items. Soccer was chosen because we assumed that soccer is a sports activity performed by a considerable portion of the respondents and, even 
Table 2. Wording of the attitude statements in each thematic section

\section{Attitude statements}

Environmental protection activities

1st item pair:

Specific "I pay attention to act and to live environmentally friendly, even though it is sometimes quite difficult for me"

General "Everybody has to be absolutely concerned about the protection of the environment"

2nd item pair:

Specific "For me personally, it is rather difficult to do something to save the environment" (reversed)

General "Everybody is responsible for the causes of environmental problems on our planet"

3rd item pair

Specific "I can imagine well participating in projects that deal with environmental protection issues (e.g., volunteering)"

General "Environmental protection is an important topic to me"

4th item pair

Specific "I am actively involved in saving the environment and, thus, make my personal contribution to the environment and its protection"

General "People behave in general very environmentally friendly" Sports activities

Specific "Soccer is a very trendy sport"

"I think soccer is one of the most popular sports all over the world"

"Soccer is my favorite sport"

General "Sport is fun"

"I can imagine well participating in a soccer contest"

"Living without sports is unhealthy"

"People do not exercise enough"

"Sport plays a central role in my life"

if this is not the case, they would encounter it on a regular basis (e.g., on television) due to its popularity in Germany. This should allow them to report a personal attitude about the topic that is relatively stable. Respondents had to answer these statements on a five-point answer scale ranging from "I strongly agree" to "I strongly disagree".

\subsubsection{Order of Filter Questions in Combination with Attitude Judgments}

The impact of questions asking about actual behavior on attitude statements was tested by varying their order as a function of questionnaire version. For example, questions such as "Are you actively involved in saving the environment?", if yes, "How do you do that?", which were presented with different response alternatives (see above), were presented either before or after the attitude statements in each thematic section of the questionnaire.

\subsection{Procedure}

The study was administered via paper-and-pencil questionnaires. Each participant received a questionnaire that had to be filled out individually under supervision of a 
teacher. Participants were told that we were interested in their personal opinions and behaviors concerning two different topics, environmental protection and sports activities, to gain better insight into their individual attitudes. Special care was taken to ensure that participants sitting next to each other received the same questionnaire version to ensure that they did not realize that two versions of one questionnaire were being applied. The whole procedure took approximately 15 minutes. All of the respondents received some sweets after completion of the survey and were informed about the aim of the study and the usage of two questionnaire versions in which question format and context were varied. None of them reported that they had become aware of the two different versions while filling out the questionnaires.

\section{Results}

All data were first analyzed for main effects of question format and context across both age groups. In addition, we looked for answer differences as a function of age group. Due to the differences between the questions across the two thematic sections, data were analyzed separately for each topic.

\subsection{Open vs. Closed Response Format}

In the section about environmental protection activities, the question that was presented either in a closed or in an open response format was preceded by a filter question that asked about active engagement in these kinds of activities. The data showed that a considerable number of respondents from both age groups indicated in the filter question that they were not actively involved in saving the environment (younger adolescents: $n=41$; older adolescents: $n=40$ ). Thus the number of participants who answered the following question, in which the response format was varied, was significantly reduced. More importantly, in younger adolescents, $\chi^{2}(1, n=104)=14.54, p<.001$, as well as in older adolescents, $\chi^{2}(1, n=84)=9.36, p=.002$, this decrease in the number of respondents was significantly more pronounced in the questionnaire version in which an open response format was presented (younger adolescents: $n=22$; older adolescents: $n=15$ ) compared to the other questionnaire version (younger adolescents: $n=41$; older adolescents: $n=29$ ). In order to take the unequal sample sizes into account, nonparametric tests were used to determine the impact of the respective response format on the answers of the two age groups in this section of the questionnaire.

A Mann-Whitney U-Test on the number of retrieved answers across the whole sample confirmed a significant effect of response format, $U=414.00, Z=5.89, p<.001$, $r=.57$. In line with our hypothesis, significantly more answers were given in a closed response format $(M d n=4$ (median)) compared to an open response format $(M d n=2)$. For example, when participants were asked whether they were actively involved in saving the environment and how they did that, $58.6 \%$ of them reported "paying attention to saving energy and water at home" when it was included in a list of answer alternatives, whereas only $2.7 \%$ gave an equivalent answer in the open response format.

Older adolescents $(M d n=2)$ provided more relevant answers in an open response format than younger adolescents $(M d n=1), U=85.00, Z=2.68, p=.007, r=.44$. Younger adolescents $(M d n=4)$, in contrast, showed the tendency to report more 
activities than older adolescents $(M d n=3)$ when a list of alternatives was provided, $U=443.50, Z=1.83, p=.067, r=.22$. Thus, if they answered this question at all, younger adolescents seemed to be more strongly influenced by the presented question format than older adolescents within this section of the questionnaire.

In the section about sports activities, only a small fraction of respondents from both age groups denied the filter question that asked whether they engage in sports (younger adolescents: $n=4$; older adolescents: $n=9$ ). In addition, in both age groups, the answers did not differ as a function of the presented questionnaire version, all $\chi^{2} \leq 1.24$, all $p \geq .724$. Thus, a 2 (response format: open vs. closed) $\times 2$ (age group: younger adolescents vs. older adolescents) analysis of variance (ANOVA) on the number of retrieved answers was used in order to examine answer differences as a function of presented response format and age group. A significant main effect of response format, $F(1,171)=33.23, p<.001, \eta_{\mathrm{p}}^{2}=.163$, showed that a higher number of sports activities was reported when a list of answer alternatives was provided $(M=4.84, \mathrm{SD}=3.31)$ compared to an open response format $(M=2.69, \mathrm{SD}=1.48)$. The biggest differences were found for sports activities that might also be categorized as leisure activities. For example, in an open response format only $9.2 \%$ of respondents reported skateboarding, while $28.4 \%$ picked this option when it was part of the response list. Reports differed most notably for the answer alternative "School sports in general": $60.2 \%$ of respondents chose this in the closed response format compared to $10.3 \%$ in the open response format.

Concerning answer differences between younger and older adolescents, no significant interaction between response format and age group was found, $F(1,171)=2.90, p=.091$, $\eta_{\mathrm{p}}^{2}=.017$. This indicates that the presented response format affected the answers of both younger (open response format: $M=2.72, \mathrm{SD}=1.49$; closed response format: $M=4.30$, $\mathrm{SD}=3.01$ ) and older adolescents (open response format: $M=2.65, \mathrm{SD}=1.50$; closed response format: $M=5.55, \mathrm{SD}=3.58$ ) to a similar degree. Thus no answer differences between the age groups were evident if the questions were related to sports activities, despite the fact that a very high number of response alternatives was presented in the closed response format (31 in total).

\subsection{Frequency of Answer Scales}

In order to analyze the influence of the presented answer scale and its frequency, answers from both questionnaire versions in each thematic section were coded on one dimension ranging from 1 - "Several times a day" to 7 - "Less than once a month". Within this combined scale, "Several times a week" and "Once a week" that appeared in both questionnaire versions were equally coded as well as "Less than once a week" from the high-frequency scale together with " $2-3$ times a month" from the low-frequency scale. In the section about environmental protection activities, the question that was either accompanied by a low- or a high-frequency scale was preceded by a filter question that asked whether participants sought out information about environmental protection issues. Approximately half of the respondents in each age group answered this filter question in the negative (younger adolescents: $n=44$; older adolescents: $n=51$ ). The presented questionnaire version did not affect the agreement with the filter question, neither in 
younger (high-frequency scale: $n=30$; low-frequency scale: $n=30$ ) nor in older adolescents (high-frequency scale: $n=15$; low-frequency scale: $n=18$ ), all $\chi^{2} \leq 0.45$, all $p \geq .503$. Younger adolescents, however, agreed with the filter question more often than older adolescents overall, $\chi^{2}(1, n=188)=6.30, p=.012$. Due to the resulting unequal sample sizes and the ordinal nature of the responses, nonparametric tests were used in order to determine the impact of the respective answer scale frequency on the answers of the two age groups.

A Mann-Whitney U-Test on the reported behavioral frequencies confirmed a significant difference in the expected direction as a function of answer scale frequency, $U=512.50$, $Z=4.52, p<.001, r=.47$. Respondents reported informing themselves about once a week when the high-frequency scale was presented $(M d n=4)$, whereas along the lowfrequency answer scale they reported informing themselves only about 2-3 times a month on average $(M d n=5)$. In other words, $53.4 \%$ of respondents reported that they sought information about environmental protection issues at least once a week when a highfrequency scale was presented, whereas only $27.1 \%$ gave an equivalent answer along the low-frequency answer scale. This confirms that an answer scale with high-frequency response alternatives resulted in higher estimates about behavioral frequencies than a low-frequency answer scale.

This answer difference was highly significant for younger adolescents, $U=171.50$, $Z=4.26, p<.001, r=.55$, whereas the difference among older adolescents only approached significance, $U=85.50, Z=1.86, p=.062, r=.22$ (see Table 3, upper panel). More specifically, $56.7 \%$ of the younger adolescents and $46.7 \%$ of the older adolescents reported that they informed themselves about environmental protection issues at least once a week when a high-frequency scale was presented. Only $20.0 \%$ of the younger adolescents and $38.9 \%$ of the older adolescents gave an equivalent answer along the low-frequency answer scale. This indicates that younger adolescents relied more than older adolescents on the frequency of the presented answer scale in order to estimate their behavioral frequency in this section of the questionnaire.

Within the section about sports activities, the relevant question in which the answer scale frequency was varied was preceded by the same filter question as the open versus closed response format question. As outlined above, only a small fraction of the respondents did not agree with this filter question (two additional respondents did not report their behavioral frequencies despite agreeing with the filter question). A MannWhitney U-Test on the reported behavioral frequencies as a function of presented answer scale did not show a significant difference between the two conditions, $U=3290.00$, $Z=1.52, p=.128, r=.12$. Respondents reported engaging in sports several times a week, regardless of which answer scale was presented (high-frequency scale: $M d n=3$; low-frequency scale: $M d n=3$ ). $90.7 \%$ of respondents reported doing sports at least once a week when a high-frequency answer scale was used, compared to $86.2 \%$ when low- frequency answer alternatives were presented.

No significant answer differences were found, neither for younger adolescents, $U=1100.50, Z=0.97, p=.331, r=.10$, nor for older adolescents, $U=583.00$, $Z=1.21, p=.225, r=.14$ (see Table 3, lower panel). Thus younger adolescents - much like older adolescents - seemed to estimate their behavioral frequencies relatively independently of the presented answer scale in this section of the questionnaire. 


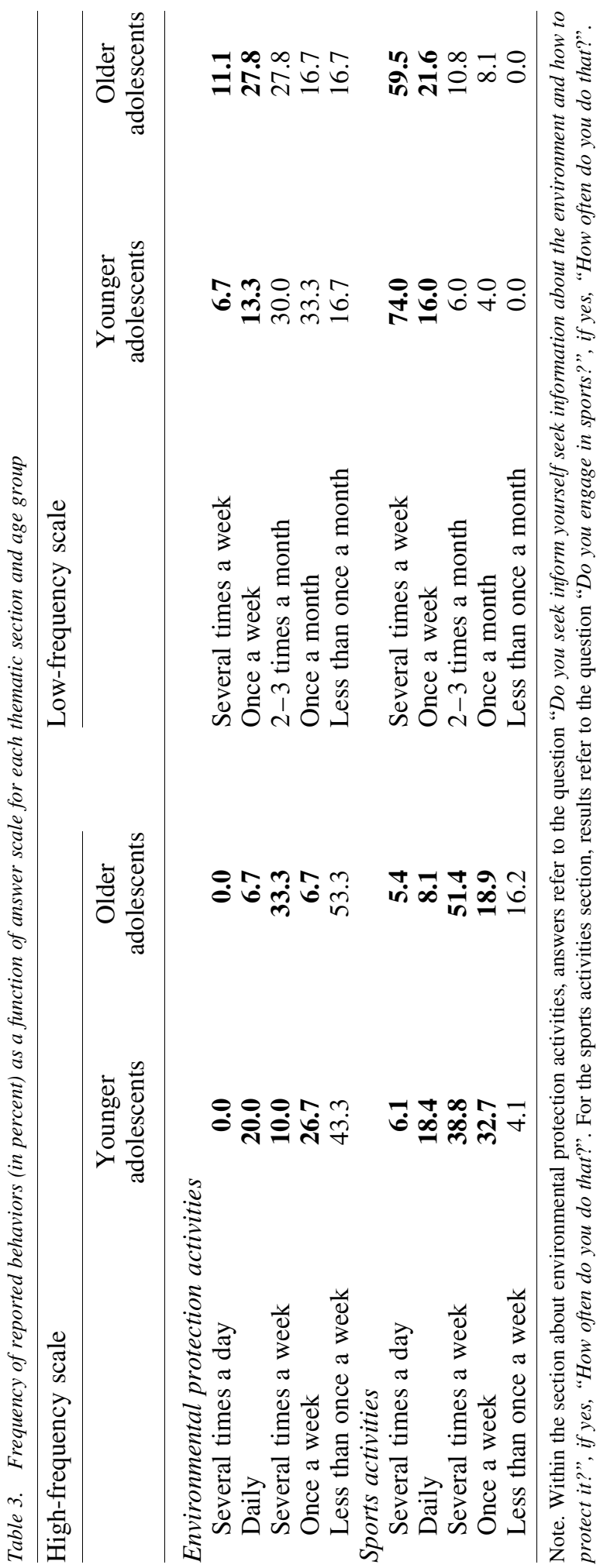




\subsection{Question Order in Attitude Judgments}

The reports about personal attitudes towards the respective topic in question were first analyzed by means of 2 (question order: specific-general vs. general-specific) $\times 2$ (age group: younger vs. older adolescents) ANOVAs to examine the respondents' level of agreement with the statements depending on their position in the questionnaire. In addition, Pearson's product-moment correlations between the item pairs were calculated for each age group in order to determine to which extent their answers were related to each other in the different conditions. Fisher r-to-z transformations were used to compare the size of the correlations (two-tailed) as a function of question order (Preacher 2002).

In the section about environmental protection activities, the agreement with the attitude statements was not influenced by the presented question order (all $F \leq 3.85, p \geq .051$, $\eta_{\mathrm{p}}^{2} \leq .020$ ). However, for seven of the eight attitude statements (except the specific item of the second item pair), significant main effects of age group were obtained, irrespective of question order or item specificity, all $F \geq 4.25, p \leq .041, \eta_{\mathrm{p}}^{2} \geq .023$. An interaction between question order and age group was not found for any of the items, all $F \leq 2.90$, $p \geq .091, \eta_{\mathrm{p}}^{2} \leq .015$. Younger adolescents generally agreed more strongly than older adolescents with the attitude statements in this section of the questionnaire. This is particularly interesting in light of the behavioral reports implying that younger adolescents did not possess much direct experience of this topic on average.

The correlational analysis yielded the following results (see Table 4, upper panel): in younger adolescents, correlations between the answers in three of the four item pairs were significantly higher when the general item had to be evaluated first. For example, the items of the third item pair correlated with $r=.28$ when the specific item "I can imagine well participating in projects that deal with environmental protection issues (e.g., volunteering)" preceded the general item "Environmental protection is an important topic to $m e$ ". The correlation increased to $r=.63$ when the general item had to be evaluated first. These results show that younger adolescents adapted their answers on statements referring to a rather subordinate/personal aspect of the topic to the more general statements in most of the cases. This pattern was not found for the second item pair, possibly due to the respective question wording. The specific item of this item pair was reversed. In older adolescents, the direction of the relationship between the item pairs was not as consistent, and the differences between the correlations as a function of presented question order were not significant. Together, the results suggest that younger adolescents, in contrast to older adolescents, provided answers that were considerably influenced by the presented question context when the topic in question was rather unfamiliar to them.

In the section about sports activities, a 2 (question order: specific-general vs. general vs. specific) $\times 2$ (age group: younger vs. older adolescents) ANOVA on the answers in each item block revealed a significant main effect of question order only for the items about sports in general, $F(1,184)=7.43, p=.007, \eta_{\mathrm{p}}^{2}=.039$. Respondents agreed more strongly with these items when they were presented after the soccer items. In addition, a significant main effect of age group was obtained for the general statements, $F(1,184)=6.95, p=.009, \eta_{\mathrm{p}}^{2}=.036$. In line with findings in the other section of the questionnaire, younger adolescents agreed more strongly with the general statements than older adolescents. 
Table 4. Pearson's Product-Moment Correlation Coefficients of the attitude statements as a function of question order for each thematic section and age group

\begin{tabular}{|c|c|c|c|c|c|c|c|}
\hline & & \multicolumn{2}{|c|}{$\begin{array}{l}\text { Specific - } \\
\text { general }\end{array}$} & \multicolumn{2}{|c|}{$\begin{array}{l}\text { General - } \\
\text { specific }\end{array}$} & \multirow[b]{2}{*}{$z$} & \multirow[b]{2}{*}{$p$} \\
\hline & & $r$ & $n$ & $r$ & $n$ & & \\
\hline \multicolumn{8}{|c|}{ Environmental protection activities } \\
\hline \multirow[t]{2}{*}{ 1st item pair } & Younger adolescents & $.39 * *$ & 51 & $.73 * * *$ & 52 & 2.55 & .011 \\
\hline & Older adolescents & $.58 * * *$ & 42 & $.52 * * *$ & 42 & 0.38 & .704 \\
\hline \multirow{2}{*}{ 2nd item pair } & Younger adolescents & -.15 & 52 & -.21 & 51 & 0.31 & .757 \\
\hline & Older adolescents & .02 & 42 & -.19 & 42 & 0.94 & .347 \\
\hline \multirow[t]{2}{*}{ 3rd item pair } & Younger adolescents & $.28^{*}$ & 52 & $.63 * * *$ & 52 & 2.25 & .024 \\
\hline & Older adolescents & $.61 * * *$ & 42 & $.36^{*}$ & 42 & 1.47 & .142 \\
\hline \multirow[t]{2}{*}{ 4th item pair } & Younger adolescents & -.03 & 52 & $.42 * *$ & 52 & 2.36 & .009 \\
\hline & Older adolescents & -.03 & 42 & .00 & 42 & 0.13 & .897 \\
\hline \multicolumn{8}{|l|}{ Sports activities } \\
\hline \multirow[t]{2}{*}{ Question blocks } & Younger adolescents & $.38 * *$ & 52 & .19 & 52 & 1.03 & .303 \\
\hline & Older adolescents & -.03 & 42 & .19 & 42 & 0.89 & .327 \\
\hline
\end{tabular}

Note. $* * *=p<.001 ; * *=p<.01 ; *=p<.05$. The results of the Fisher r-to-z transformations are shown that compare the differences between the correlation coefficients.

The correlations between the item blocks about soccer and sports in general were rather small and did not differ significantly as a function of question order, neither in younger nor in older adolescents (see Table 4, lower panel). This suggests that both age groups answered these statements fairly independently of the presented question order.

\section{4. $\quad$ Order of Filter Questions in Combination with Attitude Judgments}

To examine the impact of answering or failing to answer filter questions asking about actual engagement in environmental protection activities depending on their position in the questionnaire, only those respondents who answered the two filter questions consistently (i.e., agreed or disagreed with both filter questions) were included in the analysis. In addition, answers on each of the eight attitude statements were averaged into a composite judgment score ranging from 1 - "I strongly disagree" to 5 - "I strongly agree”. In general, respondents agreed more often with the filter questions when they preceded the attitude statements (agreed: $n=41$; disagreed: $n=20$ ) compared to the reversed question order (agreed: $n=28$; disagreed: $n=37), \chi^{2}(1, n=126)=7.40$, $p=.007$. This seemed to be mainly due to the younger adolescents, who agreed more often with the filter questions when they were presented in first (agreed: $n=27$; disagreed: $n=8$ ), but did not show any answer preference if they had already reported their attitudes about the topic in question (agreed: $n=18$; disagreed: $n=18), \chi^{2}(1, n=71)=5.63$, $p=.018$. This might have been related to the respective response format (i.e., closed response format and a high-frequency answer scale) that was used for the two filter questions in this questionnaire version. Older adolescents, in contrast, appeared to be less affected by the presented order of the filter questions (agreed before: $n=14$; disagreed before: $n=12$; agreed afterwards: $n=10$; disagreed afterwards: $n=19$ ), $\chi^{2}(1, n=55)=2.09, p=.148$. Due to the unequal sample sizes in the different 
conditions, nonparametric tests were used in order to determine the relation between answering or failing to answer the filter questions and the attitude reports.

A Mann-Whitney U-Test confirmed that subsequent attitude reports were influenced by answering or failing to answer the filter questions beforehand, $U=168.00, Z=3.73$, $p<.001, r=.48$. Respondents who failed to answer the filter questions successfully beforehand evaluated the attitude statements more neutrally $(M d n=3.00)$ than respondents who were able to report some relevant behaviors $(M d n=3.38)$. This result indicates that information about own behaviors that became accessible through these filter questions shaped the following attitude judgments accordingly. Moreover, attitude reports were less positive when the filter questions were denied beforehand than when the attitude statements preceded the filter questions $(M d n=3.50), U=337.00, Z=3.25, p=.001$, $r=.35$. No significant answer differences were found between respondents who agreed with the filter questions beforehand and those who answered them afterwards, $U=1300.00, Z=0.21, p=.832, r=.02$.

When analyzed separately for each age group, the difference between attitude reports as a function of answering or failing to answer the filter questions beforehand was significant for younger adolescents, $U=48.00, Z=2.38, p=.017, r=.40$, as well as for older adolescents, $U=40.50, Z=2.25, p=.025, r=.44$. Younger adolescents' $(M d n=3.06)$ as well as older adolescents' $(M d n=2.94)$ attitude reports were less positive when they failed to answer the filter questions successfully compared to the situation when they were able to report some relevant behaviors beforehand (younger adolescents: $M d n=3.38$; older adolescents: $M d n=3.31$ ). The attitude reports of younger adolescents who answered the filter questions afterwards $(M d n=3.63)$ were more positive than the reports of younger adolescents who failed to answer the filter questions beforehand, $U=60.50, Z=2.55, p=.011, r=.39$, but did not differ from the reports of younger adolescents who were able to answer them before, $U=427.50, Z=0.82, p=.414$, $r=.10$. In older adolescents, attitude reports did not differ in respondents who answered the filter question positively or negatively in the first place compared to respondents who answered them afterwards $(M d n=3.25)$, all $U \geq 117.00, Z \leq 1.64, p \geq .102, r \leq .26$. Thus, younger adolescents' attitude reports seemed to be more strongly influenced by the presented question order and the information brought to mind by the failure to report any relevant behaviors.

Corresponding analyses in the section about sports activities were not conducted because only 6 of 94 respondents gave a negative answer to the filter questions about their engagement in sports activities when these questions were presented first.

\section{Discussion}

This is one of the first studies investigating the impact of question format and question context on self-reports of younger and older adolescents within different topics. Results show that contextually provided cues related to certain question characteristics, response scales, and question order may exert a considerable influence on self-reports of these age groups. Additionally, our findings indicate that in most cases, the answers of adolescents aged between 11 and 13 years were more strongly affected by the presented question format and context than those of adolescents aged between 16 and 18 years. However, 
response effects were generally less pronounced when the questions referred to sports activities, a topic of which the majority of respondents had direct experience, but were stronger in the section about environmental protection activities, a topic that seemed to be more "remote" from the respondents' field of experience. As a consequence, answer differences between younger and older adolescents were mainly found in the section about environmental protection activities.

\subsection{The Impact of Question Format and Context}

A closed response format resulted in more and different answers compared to an open response format. As expected, generating answers in an open response format appeared to be more difficult than picking an answer from a list of answer alternatives, especially for younger adolescents in the section about environmental protection activities. Results further implied that the respondents were more likely to skip this question completely in this section of the questionnaire if an open response format was presented. Thus, if possible in the context of the questionnaire, younger survey respondents might avoid questions that require more effort in order to generate an appropriate answer (cf. Borgers et al. 2000). In these cases, they may use a satisficing strategy and rely more strongly on contextual cues provided by the questionnaire (cf. De Leeuw et al. 2004). This is further supported by the results in the closed response format suggesting that younger adolescents are more likely than older adolescents to check a number of activities that appear meaningful to them. In the section about sports activities, reports differed most notably for the answer alternative "School sports in general". In line with previous research, even younger survey respondents appeared to exclude activities that the researcher may take for granted as long as this was not explicitly requested within the presented question format (cf. Schwarz 1999).

In addition, higher behavioral frequencies were reported when a high-frequency answer scale was presented as compared to a low-frequency answer scale in the section about environmental protection activities. Respondents seemed to use the presented answer scale as a frame of reference for estimating the "typical" behavioral frequency within the topic in question and classifying their own activities accordingly. This pattern was more evident in younger adolescents, who might have had difficulties in remembering relevant instances of somewhat infrequent and poorly represented behavior due to their less developed cognitive skills (cf. Fuchs 2005). However, one might also argue that younger adolescents tried to make a positive impression and more than older adolescents tended towards answers they considered socially desirable in this section of the questionnaire (cf. De Leeuw et al. 2004; Kränzl-Nagl and Wilk 2000; Reynolds 1993). In addition, younger adolescents agreed with the preceding filter question more often than older adolescents in both questionnaire versions. This might have been related to the general question format, which included a list of answer alternatives in both cases, or, in other words, contextual cues that could be used to generate an answer. Younger adolescents might rely more on such cues in order to infer the intended meaning of a given question and to form an appropriate answer in a survey.

We also found that younger but not older adolescents judged statements targeting personal opinions and attitudes differently as a function of presented question order in the 
section about environmental protection activities. In line with the norm of evenhandedness (Schuman and Presser 1981), they seemed to adjust their personal preference to normative expectations that might have been implied by the general items (containing phrases like "everybody has" or "people behave"). Thus, a lack of a stable personal attitude about the topic in question might have led to an orientation towards the general statements to infer the "appropriate" attitude in the presented context and to construct their own personal attitude accordingly. Younger adolescents generally agreed more strongly with the attitude statements than older adolescents. Whether this might be due to satisficing or whether they simply possessed a more positive attitude towards these topics cannot be answered completely within the present study. It appears more likely, however, that this might be linked to the former due to a stronger tendency to behave in a socially desirable way, especially when information from authorities is received (cf. Ceci et al. 1987; Kränzl-Nagl and Wilk 2000; Reynolds 1993; Scott 2008; Warren and Lane 1995). The results further suggested that younger adolescents had some problems with answering an item that was reversed (i.e., requiring disagreement in order to express a positive attitude) in contrast to the other items (cf. Borgers et al. 2004).

In addition, we showed that the attitude statements were judged differently depending on whether they were presented before or after certain filter questions about personal behaviors and answering or and failing to answer these questions. The awareness that no relevant behaviors could be reported obviously shaped the subsequent attitude statements. This was especially evident in younger adolescents. This age group also showed a stronger tendency to agree with the filter questions when they were presented first, in contrast to the reversed question order. Given that both filter questions incorporated a list of answer alternatives when they preceded the attitude statements, one might speculate that a list of answer alternatives encouraged younger more than older adolescents to agree with the filter questions. If they were not able to answer them despite these contextual cues, however, their agreement with the following attitude statements decreased considerably. Thus younger survey respondents might try to convey a consistent picture and reaffirm their self-concept after certain information becomes accessible in the context of a survey (cf. Martin and Harlow 1992).

In sum, the results on the influence of question order on attitude reports speak against the assumption that younger survey respondents may not recognize preceding questions as a relevant context for the interpretation of following questions due to limitations in cognitive resources (cf. Fuchs 2005). As Fuchs (2009) noted, age-related differences in self-reports cannot be completely accounted for by differences in cognitive status. According to Scott (2008), children's performance in memory tasks is similar to that of adults by the age of eleven, suggesting that the younger adolescents in the present study were able to keep in mind the information that became accessible due to preceding questions. Instead, problems of literacy, confidentiality, and context seem to play an important role when conducting survey research with these age groups (Scott 2008). The participation in a scientific survey conducted in the classroom might already have shaped their interpretation of the presented questions and their perceptions about social desirability.

One limitation of the study concerns the general questionnaire design that varied four response effects within two versions of a questionnaire. For example, some of the behavioral questions could be skipped when a filter question was answered negatively 
beforehand (e.g., "Are you actively involved in saving the environment?"). Given that these skipped questions varied another effect (e.g., open vs. closed response format), the sample size was sometimes considerably reduced. Moreover, the usage of paper-andpencil questionnaires enabled respondents to see forthcoming questions. The results indicate that the characteristics of these questions might bias responses to preceding questions in order to avoid questions that require more effort to answer (e.g., when presented in an open response format). In survey research, however, many topics are often incorporated within as few questions as possible, resulting in questionnaires similar to those used in the present study. Therefore, it may be considered an important step to elucidate possible effects of the presented question characteristics on the answers of younger respondents in a typical research survey.

\subsection{The Impact of Question Content}

Younger adolescents were more influenced by the presented question format and context than older adolescents for the majority of the tested effects in the section about environmental protection activities, but not in the section about sports activities. This suggests once more that possible differences in cognitive development are unlikely to account for the present results, also given that the sample was rather homogenous in terms of academic performance. Instead, younger adolescents might be more susceptible to these kinds of effects compared to older adolescents when they are not particularly experienced with the topic in question. In these cases, suggestibility resulting from social and motivational factors might bias their responses. For the majority of respondents from both age groups, sports activities seemed to play an important role in their lives, whereas direct experiences with environmental protection activities appeared to be much less prevalent. In line with the findings from Bickart (1992), this indicates that frequent and personally relevant behaviors are better represented in memory, which enables even younger respondents to retrieve relevant information from memory rather than construct it based on contextual cues. By contrast, if experience with the question content is not given, younger age groups in particular may be highly context sensitive, susceptible towards normative expectations, and tend towards answers they may perceive as socially desirable (cf. Borgers et al. 2000; Kränzl-Nagl and Wilk 2000; Reynolds 1993). One should note, however, that the very high frequency of relevant behaviors in the section about sports activities may have attenuated possible response effects to some extent. Thus further research is warranted in future in order to examine the influence of these response effects within topics that are regularly encountered in these age groups but differ in their degree of social desirability (however, see Fuchs 2005, who found large response scale effects in younger adolescents aged 13 to 15 when asking them to report their daily TV consumption). Due to differences between the questions in the two sections of the questionnaire that resulted from the fact that they were designed to capture the characteristics of each topic in an ecologically valid way, direct comparisons between the topics could not be implemented in the present study. Nevertheless, it still provides important insights into the way younger respondents deal with questions asked in a survey and may have important applications for improving the design of questionnaires targeted at younger age groups and the interpretation of their results. 


\subsection{Implications for Survey Research With Younger Age Groups}

The results of the present study confirm the assumptions of Lipski (2000) and Scott (2008) that for questions related to their direct field of experience, younger age groups are capable of giving answers that are less influenced by the presented question format and context. It should be noted, however, that younger respondents are likely to be "novices" in many domains. Because they are still in the process of acquiring knowledge and forming firm attitudes, they might be more susceptible to context effects on many occasions. Researchers are well advised to consider this when developing and conducting a survey in order to minimize erroneous conclusions from the obtained results. Systematic pretesting could provide the researcher with an informative basis about the way young age groups think, their level of experience with the topic, and their understanding of the question (see De Leeuw et al. 2004 and Scott 2008 for different methods). Essential cues can then be derived about the most suitable design for a particular question, for example, when to favor an open over a closed response format. One further possibility might be the usage of computer-assisted or online surveys in which only one question is visible at a time to reduce potential negative effects of open response formats on the willingness to answer a question at all. Additionally, self-reports should be validated on actual behavior or by employing multiple methods, if possible. Examining bigger samples, comparing groups which differ to a larger extent in age and developmental status, as well as testing the effects within a wider range of topics, are therefore important avenues for future research.

\section{References}

Bickart, B.A. 1992. "Question-Order Effects and Brand Evaluations: The Moderating Role of Consumer Knowledge." In Context Effects in Social and Psychological Research, edited by N. Schwarz and S. Sudman. 63-80. New York: Springer.

Bishop, G.F., R.W. Oldendick, and A.J. Tuchfarber. 1983. "Effects of Filter Questions in Public Opinion Surveys.” Public Opinion Quarterly 47: 528-546. Doi: http://dx.doi. org/10.1086/268810.

Bishop, G.F. 1987. "Context Effects on Self-Perceptions of Interest in Government and Public Affairs." In Social Information Processing and Survey Methodology, edited by H.-J. Hippler, N. Schwarz, and S. Sudman. 179-199. New York: Springer.

Borgers, N., E. de Leeuw, and J. Hox. 2000. "Children as Respondents in Survey Research: Cognitive Development and Response Quality.” Bulletin de Méthodologie Sociologique 66: 60-75. Doi: http://dx.doi.org/10.1177/075910630006600106.

Borgers, N., D. Sikkel, and J. Hox. 2004. "Response Effects in Surveys on Children and Adolescents: The Effect of Number of Response Options, Negative Wording, and Neutral Mid-Point." Quality \& Quantity 38: 17-33. Doi: http://dx.doi.org/10.1023/ B:QUQU.0000013236.29205.a6.

Ceci, S.J., D.F. Ross, and M.P. Toglia. 1987. "Suggestibility of Children's Memory: Psycholegal Implications.” Journal of Experimental Psychology: General 116: 38-49. Doi: http://dx.doi.org/10.1037/0096-3445.116.1.38.

De Leeuw, E., N. Borgers, and A. Smits. 2004. "Pretesting Questionnaires for Children and Adolescents." In Methods for Testing and Evaluating Survey Questionnaires, 
edited by S. Presser, J.M. Rothgeb, M.P. Couper, J.T. Lessler, E. Martin, J. Martin, and E. Singer. 409-429. Hoboken: John Wiley \& Sons.

Fallon, Jr., T. and M. Schwab-Stone. 1994. "Determinants of Reliability in Psychiatric Surveys of Children Aged 6-12." Journal of Child Psychology and Psychiatry 35: 1391-1408. Doi: http://dx.doi.org/10.1111/j.1469-7610.1994.tb01282.x.

Festinger, L. 1954. "A Theory of Social Comparison Processes." Human Relations 7: 117-140. Doi: http://dx.doi.org/10.1177/001872675400700202.

Fuchs, M. 2005. "Children and Adolescents as Respondents: Experiments on Question Order, Response Order, Scale Effects and the Effect of Numeric Values Associated with Response Options." Journal of Official Statistics 21: 701-725.

Fuchs, M. 2009. "The Reliability of Children's Survey Responses: The Impact of Cognitive Functioning on Respondent Behavior." In Proceedings of Statistics Canada Symposium 2008. Data Collection: Challenges, Achievements and New Directions. Ottawa: StatCan. Available at: http://www.statcan.gc.ca/pub/11-522-x/2008000/article/ 10961-eng.pdf (accessed June 2013).

Gawronski, B. and J. Cesario. 2013. "Of Mice and Men: What Animal Research Can Tell Us About Context Effects on Automatic Responses in Humans." Personality and Social Psychology Review 17: 187-215. Doi: http://dx.doi.org/10.1177/1088868313480096.

Goswami, U. 2010. The Wiley-Blackwell Handbook of Childhood Cognitive Development, 2nd ed. Oxford: Wiley-Blackwell.

Heinzel, F. 2000. "Methoden und Zugänge der Kindheitsforschung im Überblick." In Methoden der Kindheitsforschung: Ein Überblick über Forschungszugänge zur kindlichen Perspektive, edited by F. Heinzel. 21-35. Weinheim: Juventa.

Kane, E. and H. Schuman. 1991. "Open Survey Questions as Measures of Personal Concern with Issues: A Reanalysis of Stouffer's Communism, Conformity, and Civil Liberties." In Sociological Methodology, edited by P. Marsden. 81-96. Oxford: Basil Blackwell.

Knäuper, B., N. Schwarz, D. Park, and A. Fritsch. 2007. “The Perils of Interpreting Age Differences in Attitude Reports: Question Order Effects Decrease with Age." Journal of Official Statistics 23: 515-528.

Kränzl-Nagl, R. and L. Wilk. 2000. "Möglichkeiten und Grenzen standardisierter Befragungen unter besonderer Berücksichtigung der Faktoren soziale und personale Wünschbarkeit." In Methoden der Kindheitsforschung: Ein Überblick über Forschungszugänge zur kindlichen Perspektive, edited by F. Heinzel. 59-75. Weinheim: Juventa.

Krosnick, J.A. 1991. "Response Strategies for Coping With the Cognitive Demands of Attitude Measures in Surveys.” Applied Cognitive Psychology 5: 213-236. Doi: http:// dx.doi.org/10.1002/acp.2350050305.

Krosnick, J.A. 1999. "Survey Research.” Annual Review of Psychology 50: 537-567. Doi: http://dx.doi.org/10.1146/annurev.psych.50.1.537.

Krosnick, J.A. and D.F. Alwin. 1987. "An Evaluation of a Cognitive Theory of ResponseOrder Effects.” Public Opinion Quarterly 51: 201-219. DOI: http://dx.doi.org/10. 1086/269029.

Lipski, J. 2000. "Zur Verlässlichkeit der Angaben von Kindern bei standardisierten Befragungen." In Methoden der Kindheitsforschung: Ein Überblick über 
Forschungszugänge zur kindlichen Perspektive, edited by F. Heinzel. 77-86. Weinheim: Juventa.

Livingstone, S., L. Haddon, and A. Gorzig. 2012. Children, Risk and Safety Online: Research and Policy Challenges in Comparative Perspective. Bristol: The Policy Press. Martin, L.L. and T.F. Harlow. 1992. "Basking and Brooding: The Motivating Effects of Filter Questions in Surveys." In Context Effects in Social and Psychological Research, edited by N. Schwarz and S. Sudman. 81-96. New York: Springer.

McNeal, J.U. 1992. Kids as Customers: A Handbook of Marketing to Children. New York: Lexington Books.

Preacher, K.J. 2002. Calculation for the Test of the Difference Between Two Independent Correlation Coefficients [Computer software]. Available at: http://quantpsy.org. (accessed June 2013).

Reynolds, W.M. 1993. "Self Report Methodology." In Handbook of Child and Adolescent Assessment, edited by T.H. Ollendick and M. Hersen. 98-120. Boston: Allyn \& Bacon.

Schuman, H. and S. Presser. 1981. Questions and Answers in Attitude Surveys: Experiments on Question Form, Wording, and Context. New York: Academic Press.

Schwarz, N. 1999. "Self-Reports: How the Questions Shape the Answers." American Psychologist 54: 93-105. Doi: http://dx.doi.org/10.1037/0003-066X.54.2.93.

Schwarz, N. 2003. "Self-Reports in Consumer Research: The Challenge of Comparing Cohorts and Cultures." Journal of Consumer Research 29: 588-594. Doi: http://dx.doi. org/10.1086/346253.

Schwarz, N. and J. Bienias. 1990. "What Mediates the Impact of Response Alternatives on Frequency Reports of Mundane Behaviors?” Applied Cognitive Psychology 4: 61-72. Doi: http://dx.doi.org/10.1002/acp.2350040106.

Schwarz, N., H. Bless, F. Strack, G. Klumpp, H. Rittenauer-Schatka, and A. Simons. 1991. "Ease of Retrieval as Information: Another Look at the Availability Heuristic." Journal of Personality and Social Psychology 61: 195-202. Doi: http://dx.doi.org/10.1037/ 0022-3514.61.2.195.

Schwarz, N. and G. Bohner. 2001. "The Construction of Attitudes." In Blackwell Handbook of Social Psychology: Intraindividual Processes, edited by A. Tesser and N. Schwarz. 436-457. Oxford: Blackwell.

Schwarz, N. and H.J. Hippler. 1995. "Subsequent Questions May Influence Answers to Preceding Questions in Mail Surveys.” Public Opinion Quarterly 59: 93-97. Doi: http://dx.doi.org/10.1086/269460.

Schwarz, N., H.J. Hippler, B. Deutsch, and F. Strack. 1985. "Response Scales: Effects of Category Range on Reported Behavior and Comparative Judgments." Public Opinion Quarterly 49: 388-395. Doi: http://dx.doi.org/10.1086/268936.

Schwarz, N. and D. Oyserman. 2001. "Asking Questions About Behavior: Cognition, Communication, and Questionnaire Construction.” American Journal of Evaluation 22: 127-161. Doi: http://dx.doi.org/10.1177/109821400102200202.

Scott, J. 2008. "Children as Respondents: The Challenge for Quantitative Methods." In Research with Children: Perspectives and Practices, edited by P. Christensen and A. James. 87-108. London: Routledge. 
Strack, F. 1992. “'Order Effects' in Survey Research: Activation and Information Functions of Preceding Questions." In Context Effects in Social and Psychological Research, edited by N. Schwarz and S. Sudman. 23-34. New York: Springer.

Strack, F. 1994. Zur Psychologie der standardisierten Befragung: Kognitive und Kommunikative Prozesse. Berlin: Springer.

Strack, F. and N. Schwarz. 2007. "Asking Questions: Measurement in the Social Sciences." In Psychology's Territories: Historical and Contemporary Perspectives from Different Disciplines, edited by M. Ash and T. Sturm. 225-250. Mahwah, NJ: Erlbaum.

Tourangeau, R. and K.A. Rasinski. 1988. "Cognitive Processes Underlying Context Effects in Attitude Measurement.” Psychological Bulletin 103: 299-314.

Tourangeau, R., K.A. Rasinski, N. Bradburn, and R. D’ Andrade. 1989. "Carryover Effects in Attitude Surveys.” Public Opinion Quarterly 53: 495-524. Doi: http://dx.doi.org/10. 1086/269169.

Warren, A.R. and P. Lane. 1995. "Effects of Timing and Type of Questioning on Eyewitness Accuracy and Suggestibility." In Memory and Testimony in the Child Witness, edited by M.S. Zaragoza, J.R. Graham, G.C.N. Hall, R. Hirschman and Y.S. Ben-Porath. 44-60. Thousand Oaks: Sage Publications.

Received April 2014

Revised April 2015

Accepted August 2015 